\title{
openheart Cardiovascular disease in HIV patients: from bench to bedside and backwards
}

\author{
Enrico Cerrato, ${ }^{1,2}$ Andrea Calcagno, ${ }^{3}$ Fabrizio D'Ascenzo, ${ }^{1}$ \\ Giuseppe Biondi-Zoccai, ${ }^{4}$ Massimo Mancone, ${ }^{4}$ Walter Grosso Marra, ${ }^{1}$ \\ Daniela Demarie, ${ }^{5}$ Pierluigi Omedè, ${ }^{1}$ Antonio Abbate, ${ }^{6}$ Stefano Bonora, ${ }^{3}$ \\ James J DiNicolantonio, ${ }^{2}$ Vicente Estrada, ${ }^{7}$ Javier Escaned, ${ }^{8}$ Claudio Moretti, ${ }^{1}$ \\ Fiorenzo Gaita ${ }^{1}$
}

To cite: Cerrato $\mathrm{E}$, Calcagno A, D'Ascenzo F, et al. Cardiovascular disease in HIV patients: from bench to bedside and backwards. Open Heart 2015;2:e000174. doi:10.1136/openhrt-2014000174

$\mathrm{EC}$ and $\mathrm{AC}$ contributed equally to this paper.

Received 14 August 2014 Revised 2 March 2015 Accepted 4 March 2015

For numbered affiliations see end of article.

Correspondence to Dr Enrico Cerrato; enrico.cerrato@gmail.com

\section{ABSTRACT}

HIV patients are exposed to a higher risk of adverse cardiovascular events, due to complex interactions between traditional risk factors and HIV infection itself in terms of ongoing endothelial dysfunctional immune activation/inflammation and increased risk of thrombosis. On the other hand, long-span antiretroviral therapy administration still raises questions on its longterm safety in an era in which life expectancy is becoming longer and longer while treatment of nonHIV-related serious events is increasingly raising concern. In this article, we will critically analyse the current knowledge of pathological and clinical aspects pertaining to the increased risk of cardiovascular events associated with HIV.

\section{INTRODUCTION}

Since the introduction of highly active antiretroviral therapy (HAART) for HIV, the decline in morbidity and mortality has been troubled by an increasing number of metabolic derangements, including dyslipidaemia, insulin resistance, abnormalities of glucose metabolism and changes in fat distribution. ${ }^{1}$ All these changes occur simultaneously with HIV infection, increasing the risk of cardiovascular (CV) disease. On the other hand, the long-span antiretroviral (ARV) therapy administration (such as abacavir or protease inhibitors (PIs)) still raises questions on its long-term safety in an era in which life expectancy is becoming longer and longer while treatment of non-HIV-related serious events is increasingly raising concern. ${ }^{2}$

A recently published meta-analysis, ${ }^{3}{ }^{4}$ including observational and randomised controlled trials (RCTs), reported the occurrence of CV disease (acute coronary syndrome (ACS) and stroke) among HIV-positive adults. In comparison to the HIV-negative population, the relative risk (RR) of events was higher both for not-HAART-treated as well as for HAARTtreated patients $(\mathrm{RR}=1.61$ (1.43 to 1.81$)$ and
$R R=2.00$ (1.70 to 2.37), respectively, all $95 \%$ CI). Moreover, the RR was higher for PI-based therapies versus non-PI treatments $(\mathrm{RR}=1.41$, $95 \%$ CI 1.20 to 1.65 ).

Two large, prospective observational cohort studies on HIV-infected patients (the HIV Outpatient Study (HOPS) ${ }^{5}$ and Data collection on Adverse events of Anti-HIV Drugs $(\mathrm{DAD})^{1}$ trials) were conducted between 1996 and 2008. The HOPS showed that death rates gradually declined from 7 per 100 person-years in 1996, to 2 deaths per 100 person-years after 1999 . At the beginning of the new century, the introduction of novel ARV therapies led to a twofold increase in HAART utilisation with about $78 \%$ of patients treated beyond the end of the millennium (figure 1). Moreover, the same studies highlighted the growing importance of heart disease in the last decade, parallel to a longer life expectancy. Figure 2 depicts the increasing incidence of $\mathrm{CV}$ death as compared with AIDS-related death. In the last decade, the ratio between these leading causes of mortality has appeared to plateau, as confirmed recently in the DAD trial ${ }^{2}(\mathrm{CV}$ Death/HIV Death ratio=0.39). This phenomenon can probably be attributed to the common event of late presentation to care. In this context, the complex interaction between traditional risk factors, comorbidities, drugs and HIV translocation still remains to be understood (figure 3). Thus, in this article, we will critically analyse the current knowledge of pathological and clinical aspects pertaining to the increased risk of $\mathrm{CV}$ events associated with HIV.

\section{HIV and the CV system}

Although the mechanism actually remains a matter of debate, HIV infection has been shown to increase the risk of coronary events, although it is very difficult to 


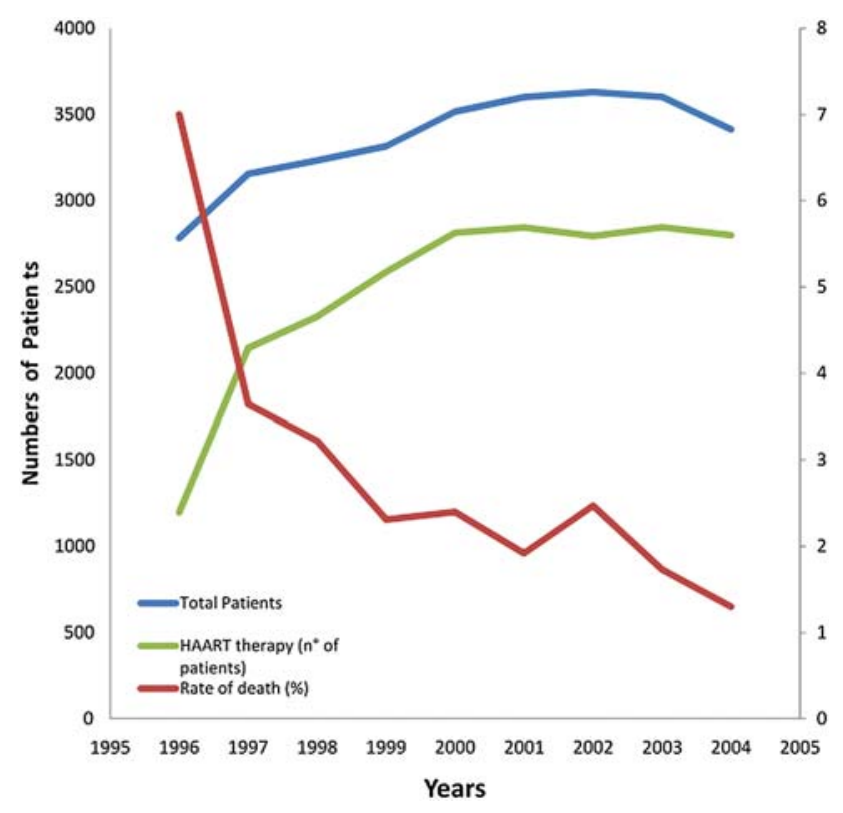

Figure 1 Numbers of patients, rate of death (\%) and numbers of patients on highly active antiretroviral therapy (HAART) in the HIV Outpatient Study (HOPS) trial, ${ }^{4}$ between 1996 and 2004.

separately appraise the role of the virus and of the therapy. ${ }^{3}$ Among 4159 HIV-positive subjects included in the Keiser Permanente database between 1996 and $2001,{ }^{6}$ the hospitalisation rate for CAD as well as the incidence of acute myocardial infarction (AMI) were significantly higher than in HIV-negative participants $(6.5 \%$ vs $3.8 \%, \mathrm{p}=0003 ; 4.3 \%$ vs $2.9 \%, \mathrm{p}=0.07$ respectively). The larger cohort study ${ }^{7}$ of almost 4000 HIV-infected patients and more than 1 million controls reported AMI in $189 \mathrm{HIV}$ and 26142 non-HIV patients with an increased rate of AMI per 1000 person-years in

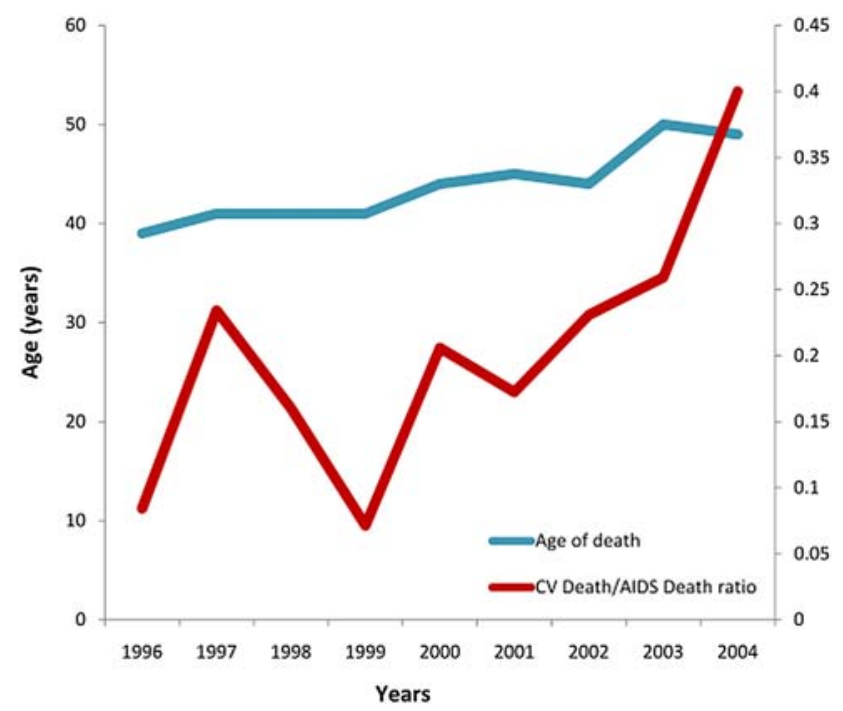

Figure 2 Cardiovascular (CV)-related/AIDS-related death ratio and age of death in the HIV Outpatient Study (HOPS) trial, ${ }^{4}$ between 1996 and 2004 patients with HIV versus those without HIV $(11.13 \%$ (95\% CI $9.58 \%$ to 12.68$)$ vs $6.98 \%$ (95\% CI $6.89 \%$ to $7.06 \%)$ ) even after adjusting for age, gender, race, hypertension, diabetes and dyslipidaemia. HIV-associated dyslipidaemia, endothelial damage/dysfunction, inflammation and hypercoagulability, which have been proposed as potential causative mechanisms of increased risk of events.

\section{Traditional CV risk factors in HIV patients}

In the cohort described by Triant et $a l^{7}{ }^{7}$ a significantly higher prevalence of hypertension $(21.2 \%$ vs $15.9 \%)$, diabetes $(11.5 \%$ vs $6.6 \%)$ and dyslipidaemia $(23.3 \%$ vs $17.6 \%)$ in HIV-infected patients than the non-HIV cohort $(\mathrm{p}<0.0001$ for each comparison) was reported. Data about the prevalence of hypertension in patients with HIV are conflicting, showing a traditional relationship with dyslipidaemia and initial kidney disease, but also an unclear causal association with HAART. Furthermore, HIV-infected men have been recognised to present a higher prevalence of smoking. On the basis of the data from the French database, ${ }^{1}$ by comparison with the general population, the sex-standardised and age-standardised morbidity ratio was estimated as 1.5 (95\% CI 1.3 to 1.7$)$ overall, 1.4 (95\% CI 1.3 to 1.6$)$ in men and $2.7(95 \%$ CI 1.8 to 3.9$)$ in women. The increase may be not only due to classical risk factors but also to exposure to combined ARV treatment, as shown by Obel et $a l^{8}$ Furthermore, gender differences have been widely reported in HIV-positive patients and they may be related to body weight, risk factors and genetic differences (several intranuclear receptors involved in cholesterol metabolism are modulated by sexual hormones): it is accepted that women have a higher risk of side effects under ARV treatment. Specifically, given the usually lower body mass index in females, several drugs may reach higher plasma concentrations potentially associated with toxicity. ${ }^{9}$

The same critical interactions between immunosuppression and drugs seem to play a crucial role in development of diabetes mellitus. Recently, Boccara et $a l^{10}$ confirmed these observations by reporting the first occurrence of ACS in HIV-infected patients at a mean age of 50 years; and male gender and tobacco-smoking participants as the most prevalent coronary risk factors. They also reported a much higher proportion of HIV-infected patients using illicit drugs compared with HIV-uninfected patients $(23 \%$ vs $6 \% ; \mathrm{p}=0.001)$. In a recent meta-analysis performed by our group, we reported an unexpectedly high incidence of traditional CV risk factors for such a young population with the exception of a low prevalence of diabetes (hypertension $22.3 \%$ (14.3\% to $30.3 \%$ ); dyslipidaemia $42.5 \%$ (33.4\% to $51.6 \%$ ); hypertriglyceridaemia $45.0 \%(23.7 \%$ to $66.4 \%)){ }^{11}$

Obesity can be highly prevalent in well-controlled HIV-positive patients since viral factors and ARVs may both affect fat metabolism. Particularly, visceral fat accumulation is common, and appropriate managing 


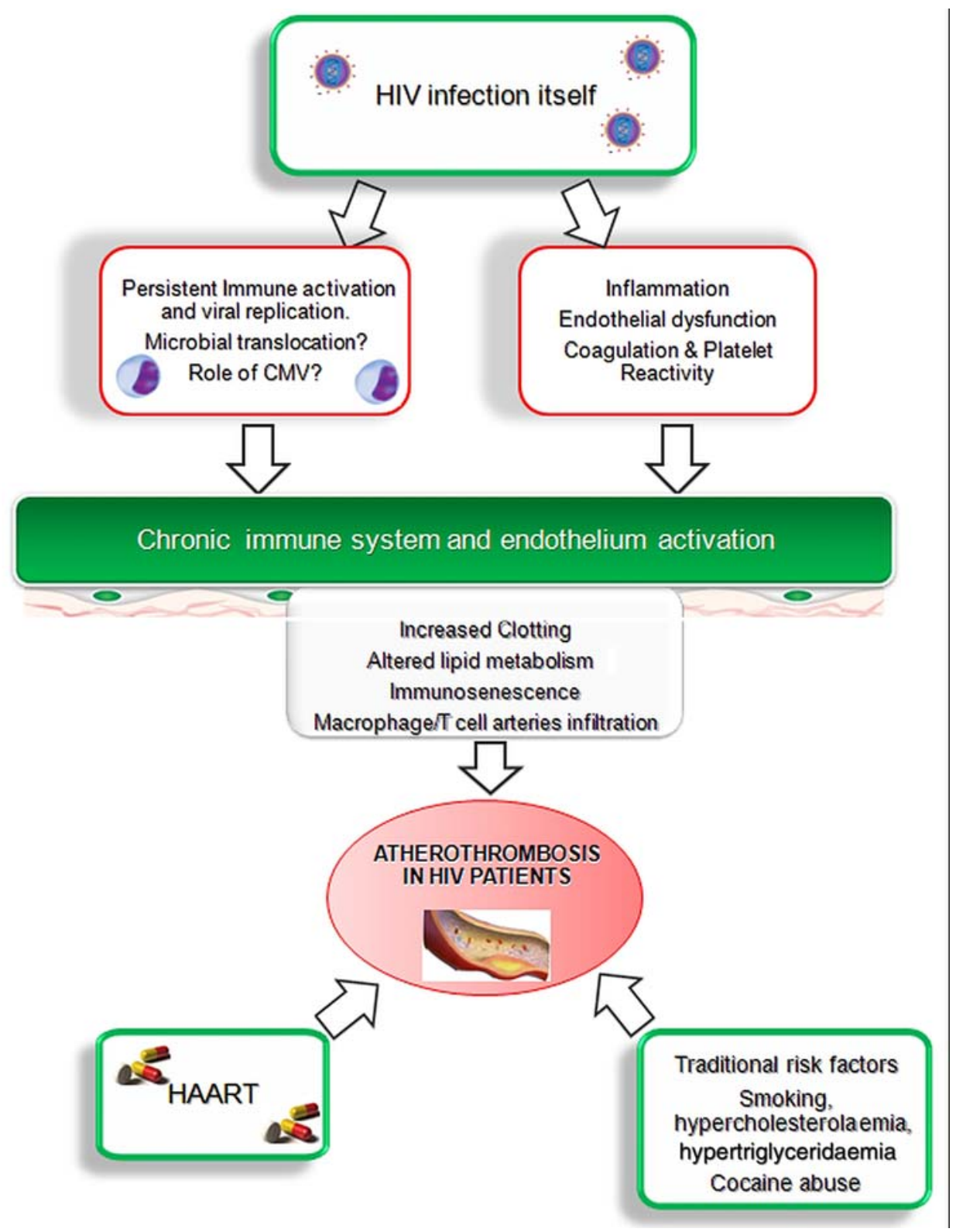

Figure 3 Pathogenesis of atherothrombosis in HIV infection. As shown in the figure, multiple pathways play a role in the development of the persistent inflammatory status. CMV, cytomegalovirus; HAART, highly active antiretroviral therapy.

strategies are warranted given its effect on CV risk. Furthermore, fat accumulation is a systemic metabolic abnormality and specific sites (such as epicardial adipose tissue) have been assessed as markers of CV risk in HIV-positive and HIV-negative patients. ${ }^{12}$

\section{HIV infection as a potential aetiological risk factor \\ Dyslipidaemia}

The values of total cholesterol (TC) and high-density lipoprotein-cholesterol (HDL-C) are rather low in the early stages of HIV infection. The progressive lowering of CD4 cell lymphocyte counts (ie, increase activation of the HIV, which finally destroys these cells) has been associated with a reduced clearance of low-density lipoprotein-cholesterol (LDL-C) particles, lower levels of apolipoprotein $\mathrm{B}$ and a decrease in HDL-C; the triglyceride levels may positively correlate to the degree of viraemia. As proposed by Mujawar et al, ${ }^{13}$ this mechanism derived from deregulations of intracellular lipid metabolism in HIV-infected macrophages due to the impairment of the ATP-binding cassette transporter A1-dependent cholesterol efflux. Conversely, Riddler $e t a l^{14}$ reported, from the multicentre AIDS cohort study, preseroconversion and post-HAART initiation values of TC, LDL-C and HDL-C. Before treatment, all these values substantially decreased $(-30,-12$ and $-22 \mathrm{mg} / \mathrm{dL}$, respectively) and subsequently, following HAART initiation, both TC and LDL-C increased while insubstantial change was registered in HDL-C values. These data provide some evidence that the observed increase in TC and LDL-C levels could represent, in a portion of subjects, a return to preseroconversion values. 
Histologically, the atherosclerosis process in HIV patients presented different features than in the general population. As reported by one necroscopic study, ${ }^{15}$ the vessel wall seems to be affected circumferentially with an uncommon prevalence of non-calcified elastic plaque. Similar findings were reported in studies comparing the nature of plaques in non-HIV versus HIV-infected men using coronary CT angiography. ${ }^{16}$ Additionally, postmortem examination studies have shown a high percentage of premature and significant atherosclerosis in young adults and children even before the introduction of PI therapy. ${ }^{15}$

\section{Endothelial dysfunction}

HIV infection and its complex interaction with the immune system plays a crucial role leading to increased cytokine expression and vascular damage. In untreated (naive) HIV-infected patients, increased plasma markers of endothelial dysfunction (ICAM1, ICAM2, ICAM3 and VCAM1) are already high at diagnosis, without significant changes during follow-up. ${ }^{10}$ In a longitudinal study, ${ }^{17}$ participants receiving HAART showed a reduction in markers of endothelial activation (VCAM1, von Willebrand factor) that was significantly associated with plasma HIV RNA levels. Moreover, endothelial function is also strictly connected with the development of early atherosclerosis and has been reported to be reduced even in young HIV-infected people under treatment when compared with those who are non-infected. ${ }^{18}$ An especially impaired endothelial function was reported in a case of PI regimen administration, probably secondary to an altered lipid metabolism. ${ }^{19}$ Notably, Flammer et al conducted a small RCT $(n=39)$ to clarify if switching from other PIs to the less atherogenic atazanavir could improve the endothelial function evaluated as flowmediated dilation (FMD) of the brachial artery. They reported no beneficial impact on vascular endothelium after switching to an unboosted atazanavir-containing combination of HAART despite a significant improvement of lipid profile in this arm. Besides, in the MONARCH RCT, ${ }^{20}$ switching from triple combination treatment (darunavir/ritonavir plus two nucleoside reverse transcriptase inhibitors (NRTIs) to darunavir/ ritonavir monotherapy) did not translate into significant changes in terms of FMD when regarding endothelial precursor or circulating endothelial cells. Conversely, data reported by the AIDS Clinical Trials Group Study in $2008^{21}$ pointed in the opposite direction, reporting a similar improvement in endothelial function (FMD) in all three treatment-naive groups randomly assigned to receive class-sparing ARV therapy regimens. Among these 82 participants, the effect on FMD appeared after 4 weeks, and persisted at 24 weeks even after adjusting for haemodynamic changes or lipid levels. However, as the authors stated, it is not possible to exclude the fact that longer and uncontrolled lipid alterations following long-term HAART ultimately may lead to negative changes in endothelial activity, and thus potentially expose participants to a higher CV risk.

\section{Inflammation}

Inflammation is associated with endothelial dysfunction in treated as well as untreated HIV-positive patients. Accelerated atherosclerosis in HIV infection can occur in the absence of HAART or detectable viraemia, or manifest immunodeficiency. High sensitivity $\mathrm{C}$ reactive protein (hsCPR) levels have been shown to be elevated in patients with HIV compared with healthy participants, and increased hsCPR levels were also associated with a higher RR of AMI. ${ }^{22}$ Hsue et $a t^{23}$ reported on carotid intima media thickness and levels of hsCRP in two similar cohorts of HIV-positive versus HIV-negative participants, revealing a greater value in all HIV patient groups, irrespective of the level of viraemia or ARV therapy even after adjustment for traditional risk factors ( $p=0.003$ ). Furthermore, CRP levels remained elevated in HIV patients. These data suggest that persistent inflammation may represent the aetiology for early atherosclerosis in these patients. Several investigations have shown that despite the control of HIV replication below the assay threshold (20, 40 or 50 copies $/ \mathrm{mL})$, HIV replication (or release from sanctuary sites) persists along with immune activation. ${ }^{24}$ Such a chronic and persistent status, possibly related to viral intestinal damage and microbial translocation, has been associated with an increase in soluble markers such as interleukin-6 (IL-6) and D-dimer. ${ }^{25}$ Therefore, HIV chronic infection can be associated with a proinflammatory status that can contribute to plaque formation and damage. Also, a relationship between hsCPR levels and the risk of cardiac dysfunction in HIV patients has been described, highlighting the role of inflammation, as a consequence of HIV virions and their harmful effect on cardiomyocytes, partly by the local release of cytokine. ${ }^{26} 27$ The ongoing inflammation probably arises from different mechanisms including (1) the evolving HIV production (if not HIV replication); (2) the co-pathogen load of cytomegalovirus (CMV) and herpes viruses; (3) the loss of immune-regulatory T cells; (4) the translocation of lipopolysaccharide across a damaged gut mucosa; and (5) the irreversible fibrosis of the lymphoid infrastructure. CMV co-infection leads to sustained antigenic stimulation with potential development of an expanded population of well-differentiated, apoptosis-resistant, senescent $\mathrm{T}$ cells limiting the immune system in recognised and challenged novel immune triggers. Recent data in a large Italian cohort $^{28}$ report that CMV/HIV co-infection was associated with the risk of non-AIDS events/deaths (including CV events) independently of other prognostic factors, supporting a potential role of CMV infection in vascular/ degenerative organ disorders commonly associated with chronic immune activation and ageing.

CMV can promote changes in endothelial cells corresponding to abnormal growth and pathogenesis of atherosclerosis promoted by proangiogenic factors 
including IL-6 and granulocyte macrophage colony stimulating factor. In a recent review, ${ }^{29}$ the authors concluded that the relationship between CMV and atherosclerosis may reflect a complex interplay between viral and immunological activation, culminating in cyclical growth, damage and repair of endothelial cells. Therefore, closer investigation of the relationship between CMV and age-related morbidities emerging in chronic HIV infection appears warranted.

\section{ARV therapy: solution or additive risk factor for CV disease?}

In the past 10 years, several studies have addressed the question of a possible association between myocardial infarction and HAART, with controversial results. ${ }^{2} 3031$ This heterogeneity arises from study design (observational cohort studies vs prospective randomised clinical trials), different populations (age, CV risk factor, previous exposure to ARV treatment) and also from different outcome definitions. One of the most relevant and large studies (the DAD) ${ }^{1}$ prospectively followed more than 20000 patients for 94469 person-years; the RR of myocardial infarction per year of PI exposure was 1.16 (1.10 to 1.23 ; CI $95 \%$ ), adjusting for hypertension, diabetes and non-nucleoside reverse transcriptase inhibitors (NNRTIs), and remained significant even after adjusting for serum lipid level.

The Strategies for Management of Antiretroviral Therapy (SMART) trial, ${ }^{32}$ one of the largest studies of HAART interruption, demonstrated that the rate of major CV events was higher if treatment was interrupted than with continuous treatment, with a HR of 1.57 (95\% CI 1.0 to $2.46, \mathrm{p}=0.05$ ). This association between treatment interruption and coronary events did not appear to be related to the level of viraemia. These findings pointed out that suppression of HIV itself plays a main part in reducing proinflammatory cytokines. Furthermore, an elevated IL-6 level was significantly associated with the development of $\mathrm{CV}$ disease $(\mathrm{OR}=2.8$, $\mathrm{p}=0.03$ ). Furthermore, in the treatment-interruption arm, IL-6 and D-dimer were significantly elevated 1 month after randomisation with a strong association with death $(\mathrm{OR}=12.6, \mathrm{p}<0.0001$ for $\mathrm{IL}-6$; $\mathrm{OR}=13.1$, $\mathrm{p}<0.0001$ for D-dimer).

\section{Protease inhibitors and metabolic complications}

Changes in body fat distribution (lipodystrophy including both lipoatrophy and lipohypertrophy) are common in HIV-infected participants and typically start to manifest after 6-12 months of ARV therapy. ${ }^{33}$ Lipoatrophy denotes a decrease in adipose tissue volume while lipohypertrophy denotes the opposite, and it commonly occurs in visceral adipose tissue and in the upper trunk. This abnormal redistribution of fat has clear clinical implications such as modifications of body image (thus affecting medication adherence), ectopic fat distribution (into the heart and liver tissues, for instance) and, eventually, increased CV risk. ${ }^{34}$ Furthermore, fat redistribution may also promote insulin resistance through altered secretion of adipokines and other inflammatory markers (such as ILs 6, 8 and 10, and macrophage chemotactic protein-1).

The HIV and HAART associated lipodystrophy is probably a multifactor in its aetiology arising from HIV infection itself, affecting both lipid/glucose metabolism and insulin sensitivity, and producing viral proteins, altering the activity of glucocorticoid receptors in several tissues.

As recently reported in a registry including more than $800 \mathrm{HIV} /$ AIDS patients, NRTI-based and PI-based regimens were associated with a high risk for developing lipohypertrophy ( $\mathrm{OR}=2.1,95 \%$ CI 1.7 to $3.3, \mathrm{p}<0.01$; $\mathrm{OR}=6.1,95 \%$ CI 4.1 to $9.7, \mathrm{p}<0.01$, respectively). ${ }^{35} \mathrm{PIs}$ have been involved in this process through several pathways that include inhibition of apolipoprotein B degradation, inhibition of insulin signalling pathways and blocking the function of the glucose transporter GLUT-4, thus diminishing insulin-stimulated glucose uptake. Conversely, thymidine analogue regimens (ie, stavudine, zidovudine) were associated with a high risk of lipoatrophy. ${ }^{36}$

\section{The abacavir controversy}

Ever since the DAD study reported an association between the use of the nucleoside reverse transcriptase inhibitor abacavir and an increase of $\mathrm{CV}$ risk in HIV-1-infected patients, there has been controversy around this drug. ${ }^{2}$ Over the years, different studies have reported disparate results: some cohort studies and a meta-analysis seemed to confirm the observation, ${ }^{30}$ whereas other recent studies and another meta-analysis seemed to contradict this hypothesis. ${ }^{31}$ Nevertheless, the major treatment guidelines ${ }^{37} 38$ warrant caution in the prescription of this drug in patients already showing a high CV risk based on traditional parameters. Nonetheless, a biological mechanism explaining the reasons for this finding has never been elucidated. Despite an initial observation of higher levels of inflammatory markers in a cross-sectional analysis of samples from two observational cohorts, subsequent longitudinal studies failed to detect significant associations between the use of abacavir and changes in the levels of $\mathrm{CV}$ risk biomarkers (prothrombin fragment $\mathrm{F}(1+2)$, D-dimer, high-sensitivity CRP, IL-8, intercellular adhesion molecule-1, vascular adhesion molecule-1, E-selectin, P-selectin, serum amyloid-A and serum amyloid-P). One of the hypotheses was the higher prevalence of chronic kidney disease in abacavir-treated patients (being the most common alternative to the nephrotoxic tenofovir), thus supporting the increased $\mathrm{CV}$ risk.

\section{Characteristics of CAD in HAART-treated patients}

As reported in previous paragraphs, for reasons not yet completely understood, HIV-infected patients on longterm treatment have a greater prevalence of atherosclerotic disease and vascular dysfunction compared with an age-matched uninfected cohort. $^{39}$ Additionally, as 
reported in cases of coronary artery disease, patients tend to manifest an illness with more aggressive characteristics, with a higher rate of ST-segment elevation myocardial infarction and multivessel involvement following the first presentation, as our group recently reported in a meta-analysis ${ }^{11}$ of 11 studies including 2442 HIV-patients presenting with ACS. These characteristics had a higher incidence than in contemporary ACS registries of non-HIV patients ${ }^{40}$ and combined together could in part explain the higher rates of in-hospital events registered for HIV patients. Conversely, other studies have reported a more favourable in-hospital outcome in absence of significant haemodynamic compromise. ${ }^{41}$ However, HIV-infected patients undergoing percutaneous coronary intervention could be potentially exposed to a high incidence of non-fatal myocardial reinfarction, restenosis and in-stent thrombosis, as a result of a prothrombotic state.

In the report of the Soweto Study Cohort, ${ }^{42} 518$ HIV-positive patients (mostly HAART-treated) admitted for a first diagnosis of CV disease between 2006 and 2008, questioned the actual incidence of coronary artery disease (CAD), reporting a relatively infrequent incidence of ACS (3\%). This finding casts doubt on the potential role of publication bias and confounding factors in overestimation of HIV and PI exposure risk. We recently published a large multicentre registry ${ }^{43}$ including patients presenting with ACS. This cohort of participants demonstrated a significantly increased risk for cardiac death in absence of therapy with NRTI as well as higher risk of AMI in cases of CD4 cell count $<200 / \mathrm{mm}^{2}$. Similar result emerged from another study recently published by Silverberg et $a l,{ }^{44}$ in which a low nadir CD4 was the only characteristic associated with AMI. Conversely, the association between low CD4 nadir and $\mathrm{CV}$ events has been observed in some but not all studies; ${ }^{4}{ }^{45}$ however, patients with a low nadir have usually been exposed to HIV for several years, have a blunted immunological recovery and have a higher incidence of HIV-associated and non-associated diseases including metabolic complications. ${ }^{46} 47$

These findings strengthen the recommendation of early HAART treatment initiation. In the occurrence of coronary syndrome, no other recommendation in HIV population was provided by current guidelines and up to now no specific trial subanalysis has been designed to specifically explore this setting. However, it seems reasonable to analyse the revascularisation strategy carefully weighing such features as, for example, the stage of HIV disease and related comorbidities, expected adherence to therapies, bleeding risk, specific drug-drug interactions (DDIs), etc. In the next paragraph, we summarised some data regarding the impact of risk factor modification and pharmacological treatment.

\section{Modification of risk factors}

The early detection and treatment of comorbidities and modifiable risk factors through lifestyle changes such as smoking cessation and dietary alterations are likely to have a significant impact on CV risk in this population. ${ }^{22}$

Even if HIV infection by itself or with HAART treatment could increase the risk of plaque rupture and atherothrombosis, routine secondary prevention does not take into account this challenge. Moreover, LDL goals are less frequently achieved in HIV-infected patients during follow-up. A recently published small randomised trial $^{48}$ involving $83 \mathrm{HIV}$ patients demonstrated that an appropriate dietary modification with a moderate reduction of fat intake (from $31 \pm 7 \%$ to $21 \pm 3 \%$ of calories) was able to prevent the increase in plasma cholesterol, LDL-C and triglycerides associated with HAART prescription in patients who were previously naïve. These interventions led to a significant reduction in dyslipidaemia after 1 year follow-up (21\% vs $68 \%$ in control group; $\mathrm{p}<0.001)$, pointing out the central role of a correct lifestyle modification in this setting. Despite this fact, a reduction in surrogate markers (lipids) does not necessarily mean that this will lead to a reduction in clinical endpoints with such a dietary intervention, also because this kind of intervention by itself has never been shown to improve survival.

Furthermore, all HIV care providers should consider smoking cessation a high priority, considering the high prevalence of this behaviour and the proven efficacy of cessation interventions in the HIV population. ${ }^{10}$

\section{Pharmacological treatment}

Dedicated guidelines for the management of hyperlipidaemia secondary to HAART have been developed by the Infectious Disease Society of America (IDSA) and Adult AIDS Clinical Trials Group (AACTG). ${ }^{49}$ These recommendations are largely based on National Cholesterol Education Program Adult Treatment Panel III (NCEP ATP III) guidelines, and advocate adjusting individual cholesterol levels through estimation of Framingham predicted 10-year CV risk. Currently, there is no difference in hyperlipidaemia target goal treatment between HIV and non-HIV patients. European Society of Cardiology/European Atherosclerosis Society 2011 lipid guidelines $^{50}$ for the first time pointed out that HIV-infected patients represent a high-risk group and need special management. However, the recommendation was not clear on the goal for LDL-C levels as there were no trials on whether statins ameliorate CV morbidity and mortality in primary prevention in this specific population. The key point to consider in the choice of specific lipid-lowering therapy is the high prevalence of DDIs in this population. Most ARV drugs have an impact on drug metabolising enzymes such as cytochrome P450 isoforms (CYP3A4, CYP2B6) and drug transporters (such as p-glycoprotein and OATP1B1) and therefore the risk of DDIs is very high. ${ }^{8}$ The most complete database on this subject is one developed and managed by the University of Liverpool (available at http://www. hiv-druginteractions.org). In general PIs (with the exception of tipranavir), there are potent CYP and OATP1B1 
inhibitors suggesting the avoidance of some lipid-lowering agents or an initial reduced dose. Therefore, the suggested agents include pravastatin (not metabolised by CYP3A4), fluvastatin (metabolised CYP2C9) and pitavastatin (minimally metabolised by CYP2C9 and glucuronidation). Rosuvastatin concentrations appear to increase when used in combination with some PIs, thus the suggested starting dose is $10 \mathrm{mg},{ }^{51}$ whereas simvastatin and lovastatin administration should be avoided due to the increased risk of rhabdomyolysis. ${ }^{52}$ Unfortunately, data concerning a possible role of statins as a primary prevention treatment are still lacking. Moreover, CV risk scores, such as Framingham and other similar systems, did not receive a specific validation for HIV patients. The only device HIV-tailored and currently available is the DAD 5 Year Estimated Risk calculator, ${ }^{53}$ however, further data on its validation are warranted.

Regarding the prescription of antiplatelet drugs, current guidelines do not provide specific recommendations in this setting. However, some data suggested a role of aspirin in primary prevention in the presence of high CV risk. ${ }^{54}$

A more complex scenario is represented by the interaction between P2Y12 inhibitors (clopidogrel, prasugrel and ticagrelor) and platelet activity in HIV-treated patients, mainly depending on specific drug-drug metabolism. The new P2Y12 agent prasugrel is bioactivated by cytochromes $\mathrm{P} 450$ (CYP) 3A/2B6. Ritonavir, a frequently prescribed PI drug, demonstrated its potential ability to block prasugrel CYP3A4 bioactivation in vitro and also in healthy male volunteers. ${ }^{55}$ Therefore, a reduction in prasugrel efficiency may be observed in patients, even if until now there is no specific recommendation for avoiding this association; whereas no data regarding ticagrelor in this setting have been reported to date.

Conclusive data regarding how to manage HAART in the setting of secondary prevention after ACS are substantially lacking. Most likely, the avoidance of PI regimens after an AMI might be a prudent. Even if the association with increased incidence of AMI was observed with cumulative exposure to lopinavir/ritonavir (and not to atazanavir/ritonavir), ${ }^{56}$ the whole class has been associated with impaired lipid and glucose metabolism. Certainly further investigation is warranted.

\section{CONCLUSION}

In the short-term, ARV therapy has been shown to reduce CV disease (although this could be simply related to short time exposition to both virus and therapies); a long-term follow-up is required to determine if any benefit (or harm) is derived. The results of ongoing trials will provide important information on how to manage the timing of HAART initiation optimising risk benefit. The Strategic Timing of Antiretroviral Treatment (START) trial $^{57}$ includes ARV-naive HIV-positive participants with CD4 counts greater than 500 cells $/ \mathrm{mm}^{3}$; it is taking place at about
90 sites in nearly 30 countries where participants are randomised to either receive ARV treatment immediately or to defer treatment until their first CD4 count is less than 350 cells $/ \mathrm{mm}^{3}$, or when they have clinical signs of advanced HIV disease. Such a randomised study will, therefore, address the question of whether the purported benefits of early therapy (as is suggested by the Department of Health and Human Services guidelines) may overcome the expected drugassociated side effects.

Other challenges and open issues remain, concerning the best time to start ARV therapy, the best regimen in patients with established $\mathrm{CAD}$, the role of antiinflammatory and antithrombotic drugs as well as the long-term clinical outcomes in HIV-positive patients, 'doomed' in the modern era to live longer and to face age-related morbidities.

In conclusion, the purpose of this work is to provide a 'snapshot' of current know-how concerning CV risk in the HIV-infected population even in absence of data from large registries or randomised clinical trials. We could not suggest any clear and conclusive recommendations to the reader on these grounds. Certainly, in our opinion, only synergic cooperation among different specialists can be the keystone to better individualisation of treatment in the chronic and in the acute setting.

\section{Author affiliations}

${ }^{1}$ Division of Cardiology, University of Turin, Turin, Italy (www.cardiogroup. org)

${ }^{2}$ Wegmans Pharmacy, Ithaca, New York, USA

${ }^{3}$ Division of Infectious Disease, Amedeo di Savoia Hospital, Turin, Italy

${ }^{4}$ Department of Medico-Surgical Sciences and Biotechnologies, Sapienza University of Rome, Latina, Italy

${ }^{5}$ Cardiology Department, Maria Vittoria Hospital, Turin, Italy

${ }^{6}$ VCU Pauley Heart Center, Richmond, Virginia, USA

${ }^{7}$ Infectious Disease Department, Hospital Clinico San Carlos, Madrid, Spain

${ }^{8}$ Interventional Cardiology, Hospital Clinico San Carlos, Madrid, Spain

Acknowledgements The authors would like to thank Matteo Victor Parmigiani for his expert assistance in the revision of the manuscript.

Contributors $E C, A C, S B$ and $C M$ were involved in conception and design. $E C$, $A C, G B-Z$ and $A A$ were involved in acquisition of data. MM, DD, WGM and AA were involved in analysis and interpretation of data. EC, AC, FD, WGM and PO were involved in drafting of the manuscript. $F D, G B-Z, M M, D D, W G M, A A$, JJD, VE, JE, CM and FG were involved in critical revision of the manuscript. $\mathrm{VE}, \mathrm{JE}, \mathrm{CM}$ and $\mathrm{FG}$ were involved in supervision of the manuscript.

Competing interests None declared.

Provenance and peer review Not commissioned; externally peer reviewed.

Data sharing statement No additional data are available.

Open Access This is an Open Access article distributed in accordance with the Creative Commons Attribution Non Commercial (CC BY-NC 4.0) license, which permits others to distribute, remix, adapt, build upon this work noncommercially, and license their derivative works on different terms, provided the original work is properly cited and the use is non-commercial. See: http:// creativecommons.org/licenses/by-nc/4.0/

\section{REFERENCES}

1. Smith C, Sabin CA, Lundgren JD, et al., Data Collection on Adverse Events of Anti-HIV drugs (D:A:D) Study Group. Factors associated 
with specific causes of death amongst HIV-positive individuals in the D:A:D Study. AIDS 2010;24:1537-48.

2. Rhew DC, Bernal M, Aguilar D, et al. Association between protease inhibitor use and increased cardiovascular risk in patients infected with human immunodeficiency virus: a systematic review. Clin Infect Dis 2003;37:959-72.

3. Islam FM, Wu J, Jansson J, et al. Relative risk of cardiovascular disease among people living with HIV: a systematic review and meta-analysis. HIV Med 2012;13:453-68.

4. Freiberg MS, Chang $\mathrm{C}-\mathrm{CH}$, Kuller $\mathrm{LH}$, et al. HIV infection and the risk of acute myocardial infarction. JAMA Intern Med 2013;173:614-22.

5. Palella FJ, Baker RK, Moorman AC, et al., HIV Outpatient Study Investigators. Mortality in the highly active antiretroviral therapy era: changing causes of death and disease in the HIV outpatient study. J Acquir Immune Defic Syndr 2006;43:27-34.

6. Klein D, Hurley LB, Quesenberry CPJ, et al. Do protease inhibitors increase the risk for coronary heart disease in patients with HIV-1 infection? J Acquir Immune Defic Syndr 2002;30:471-7.

7. Triant VA, Lee $\mathrm{H}$, Hadigan $\mathrm{C}$, et al. Increased acute myocardial infarction rates and cardiovascular risk factors among patients with human immunodeficiency virus disease. J Clin Endocrinol Metab 2007;92:2506-12.

8. Obel N, Thomsen HF, Kronborg G, et al. Ischemic heart disease in HIV-infected and HIV-uninfected individuals: a population-based cohort study. Clin Infect Dis 2007:44:1625-31.

9. Calcagno A, Gonzalez de Requena D, Simiele M, et al. Tenofovir plasma concentrations according to companion drugs: a cross-sectional study of HIV-positive patients with normal renal function. Antimicrob Agents Chemother 2013;57:1840-3.

10. Boccara F, Mary-Krause M, Teiger E, et al., Prognosis of Acute Coronary Syndrome in HIV-infected patients (PACS) Investigators. Acute coronary syndrome in human immunodeficiency virus-infected patients: characteristics and 1 year prognosis. Eur Heart $J$ 2011;32:41-50.

11. D'Ascenzo $\mathrm{F}$, Moretti $\mathrm{C}$, Omedè $\mathrm{P}$, et al. Cardiac remote ischaemic preconditioning reduces periprocedural myocardial infarction for patients undergoing percutaneous coronary interventions: a meta-analysis of randomised clinical trials. Eurolntervention 2014:9:1463-71.

12. Guaraldi G, Scaglioni R, Zona S, et al. Epicardial adipose tissue is an independent marker of cardiovascular risk in HIV-infected patients. AIDS 2011;25:1199-205.

13. Mujawar Z, Rose H, Morrow MP, et al. Human immunodeficiency virus impairs reverse cholesterol transport from macrophages. PLoS Biol 2006;4:e365

14. Riddler SA, Smit E, Cole SR, et al. Impact of HIV infection and HAART on serum lipids in men. JAMA 2003;289:2978-82.

15. Webber BJ, Seguin PG, Burnett DG, et al. Prevalence of and risk factors for autopsy-determined atherosclerosis among US service members, 2001-2011. JAMA 2012;308:2577-83.

16. Zanni MV, Abbara S, Lo J, et al. Increased coronary atherosclerotic plaque vulnerability by coronary computed tomography angiography in HIV-infected men. AIDS 2013;27:1263-72.

17. Gresele $\mathrm{P}$, Falcinelli $\mathrm{E}$, Sebastiano $\mathrm{M}$, et al. Endothelial and platelet function alterations in HIV-infected patients. Thromb Res 2012;129:301-8.

18. Charakida $\mathrm{M}$, Donald $\mathrm{AE}$, Green $\mathrm{H}$, et al. Early structural and functional changes of the vasculature in HIV-infected children: impact of disease and antiretroviral therapy. Circulation 2005;112:103-9.

19. Stein JH, Klein MA, Bellehumeur JL, et al. Use of human immunodeficiency virus-1 protease inhibitors is associated with atherogenic lipoprotein changes and endothelial dysfunction. Circulation 2001;104:257-62.

20. Guaraldi G, Zona S, Cossarizza A, et al., Randomized trial to evaluate cardiometabolic and endothelial function in patients with plasma HIV-1 RNA suppression switching to darunavir/ritonavir with or without nucleoside analogues. HIV Clin Trials 2013;14: $140-8$.

21. Torriani FJ, Komarow L, Parker RA, et al., ACTG 5152 s Study Team. Endothelial function in human immunodeficiency virus-infected antiretroviral-naive subjects before and after starting potent antiretroviral therapy: the ACTG (AIDS Clinical Trials Group) Study 5152s. J Am Coll Cardiol 2008;52:569-76.

22. Cerrato E, D'Ascenzo F, Biondi-Zoccai G, et al. Acute coronary syndrome in HIV patients: from pathophysiology to clinical practice. Cardiovasc Diagn Ther 2012;2:50-5.

23. Hsue PY, Hunt PW, Schnell A, et al. Role of viral replication, antiretroviral therapy, and immunodeficiency in HIV-associated atherosclerosis. AIDS 2009;23:1059-67.
24. Ostrowski SR, Katzenstein TL, Pedersen BK, et al. Residual viraemia in HIV-1-infected patients with plasma viral load \&lt; or=20 copies $/ \mathrm{ml}$ is associated with increased blood levels of soluble immune activation markers. Scand J Immunol 2008;68:652-60.

25. Boulware DR, Hullsiek KH, Puronen CE, et al. Higher levels of CRP, D-dimer, IL-6, and hyaluronic acid before initiation of antiretroviral therapy (ART) are associated with increased risk of AIDS or death $J$ Infect Dis 2011;203:1637-46.

26. Cerrato E, D'Ascenzo F, Biondi-Zoccai G, et al. Cardiac dysfunction in pauci symptomatic human immunodeficiency virus patients: a meta-analysis in the highly active antiretroviral therapy era. Eur Heart J 2013;34:1432-6.

27. Cannillo M, D'Ascenzo F, Grosso Marra W, et al., Heart failure in patients with human immunodeficiency virus: a review of the literature. J Cardiovasc Med (Hagerstown) 2014. Published Online First: 23 Jul 2014.

28. Lichtner M, Cicconi P, Vita S, et al., ICONA Foundation Study. Cytomegalovirus coinfection is associated with an increased risk of severe non-AIDS-defining events in a large cohort of HIV-infected patients. J Infect Dis 2015;211:178-86.

29. Barrett L, Fowke KR, Grant MD. Cytomegalovirus, aging, and HIV: a perfect storm. AIDS Rev 2012;14:159-67.

30. Choi Al, Vittinghoff E, Deeks SG, et al. Cardiovascular risks associated with abacavir and tenofovir exposure in HIV-infected persons. AIDS 2011;25:1289-98.

31. Cruciani M, Zanichelli V, Serpelloni G, et al. Abacavir use and cardiovascular disease events: a meta-analysis of published and unpublished data. AIDS 2011;25:1993-2004.

32. El-Sadr WM, Lundgren JD, Neaton JD, et al., Strategies for Management of Antiretroviral Therapy (SMART) Study Group. CD4+ count-guided interruption of antiretroviral treatment. $N$ Engl J Med 2006;355:2283-96.

33. Guaraldi G, Stentarelli C, Zona S, et al. The natural history of HIV-associated lipodystrophy in the changing scenario of HIV infection. HIV Med 2014;15:587-94.

34. Ena J, Benito C, Llácer $P$, et al. [Abnormal body fat distribution and type of antiretroviral therapy as predictors of cardiovascular disease risk in HIV-infected patients]. Med Clínica 2004;122:721-6.

35. Dragović G, Danilović D, Dimić A, et al. Lipodystrophy induced by combination antiretroviral therapy in HIV/AIDS patients: a Belgrade cohort study. Vojnosanit Pregl 2014;71:746-50.

36. Joly V, Flandre P, Meiffredy V, et al. Increased risk of lipoatrophy under stavudine in HIV-1-infected patients: results of a substudy from a comparative trial. AIDS 2002;16:2447-54.

37. Panel on Antiretroviral Guidelines for Adults and Adolescents. Guidelines for the use of antiretroviral agents in HIV-1-infected adults and adolescents. Department of Health and Human Services, 2012:1-166. http://www.aidsinfo.nih.gov/ContentFiles/Adultan dAdolescentGL.pdf

38. European AIDS Clinical Society (EACS). Guidelines: clinical management and treatment of HIV infected adults in Europe, prevention and management of non-infectious comorbidities in HIV version 6.1. 2012. http://www.europeanaidsclinicalsociety.org

39. Hsue PY, Deeks SG, Hunt PW. Immunologic basis of cardiovascular disease in HIV-infected adults. J Infect Dis 2012;205:S375-82.

40. Jolly SS, Shenkman H, Brieger D, et al. GRACE Investigators. Quantitative troponin and death, cardiogenic shock, cardiac arrest and new heart failure in patients with non-ST-segment elevation acute coronary syndromes (NSTE ACS): insights from the Global Registry of Acute Coronary Events. Heart 2011;97:197-202.

41. Matetzky S, Domingo M, Kar S, et al. Acute myocardial infarction in human immunodeficiency virus-infected patients. Arch Intern Med 2003;163:457-60.

42. Sliwa K, Carrington MJ, Becker A, et al. Contribution of the human immunodeficiency virus/acquired immunodeficiency syndrome epidemic to de novo presentations of heart disease in the Heart of Soweto Study cohort. Eur Heart J 2012;33:866-74.

43. D'Ascenzo F, Cerrato E, Appleton D, et al. Prognostic indicators for recurrent thrombotic events in HIV-infected patients with acute coronary syndromes: use of registry data from 12 sites in Europe, South Africa and the United States. Thromb Res 2014;134:558-64.

44. Silverberg MJ, Leyden WA, Xu L, et al. Immunodeficiency and risk of myocardial infarction among HIV-positive individuals with access to care. J Acquir Immune Defic Syndr 2014;65:160-6.

45. Montlahuc C, Guiguet M, Abgrall S, et al., French Hospital Database ANRS $\mathrm{CO} 4$ cohort. Impact of late presentation on the risk of death among HIV-infected people in France (2003-2009). J Acquir Immune Defic Syndr 2013;64:197-203.

46. Serrano-Villar S, Sainz T, Lee SA, et al. HIV-infected individuals with low CD4/CD8 ratio despite effective antiretroviral therapy exhibit altered T cell subsets, heightened CD8+ T cell activation, and 
increased risk of non-AIDS morbidity and mortality. PLoS Pathog 2014;10:e1004078.

47. Grinspoon S, Carr A. Cardiovascular risk and body-fat abnormalities in HIV-infected adults. N Engl J Med 2005;352:48-62.

48. Lazzaretti RK, Kuhmmer R, Sprinz E, et al. Dietary intervention prevents dyslipidemia associated with highly active antiretroviral therapy in human immunodeficiency virus type 1-infected individuals: a randomized trial. J Am Coll Cardiol 2012;59:979-88.

49. Expert Panel on Detection, Evaluation, and Treatment of High Blood Cholesterol in Adults. Executive summary of the third report of the National Cholesterol Education Program (NCEP) expert panel on detection, evaluation, and treatment of high blood cholesterol in adults (Adult Treatment Panel III). JAMA 2001;285:2486-97.

50. Catapano AL, Reiner Z, De Backer G, et al., European Society of Cardiology (ESC), European Atherosclerosis Society (EAS). ESC/ EAS Guidelines for the management of dyslipidaemias. The Task Force for the management of dyslipidaemias of the European Society of Cardiology (ESC) and the European Atherosclerosis Society (EAS). Atherosclerosis 2011;217:3-46.
51. Busti AJ, Bain AM, Hall RG, et al. Effects of atazanavir/ritonavir or fosamprenavir/ritonavir on the pharmacokinetics of rosuvastatin. $J$ Cardiovasc Pharmacol 2008;51:605-10.

52. Hare CB, Vu MP, Grunfeld C, et al. Simvastatin-nelfinavir interaction implicated in rhabdomyolysis and death. Clin Infect Dis 2002;35: e111-12.

53. DAD 5 Year Estimated Risk calculator. http://www.cphiv.dk/tools/ dadriskequations/tabid/437/default.aspx

54. Wolff T, Miller T, Ko S. Aspirin for the primary prevention of cardiovascular events: an update of the evidence for the US Preventive Services Task Force. Ann Intern Med 2009;150:405-10.

55. Ancrenaz V, Déglon J, Samer C, et al. Pharmacokinetic interaction between prasugrel and ritonavir in healthy volunteers. Basic Clin Pharmacol Toxicol 2013;112:132-7.

56. d'Arminio Monforte A, Reiss P, Ryom L, et al. Atazanavir is not associated with an increased risk of cardio- or cerebrovascular disease events. AIDS 2013;27:407-15.

57. Strategic Timing of Antiretroviral Treatment (START). http:// clinicaltrials.gov/ct2/show/NCT00867048 